\title{
Cholesteatoma as a late complication of paraganglioma surgery \\ The role of diffusion-weighted MR imaging
}

\author{
Cecília C.B. Brito' ${ }^{1}$ Rosane S. Machado², \\ Felippe Felix³, Emerson L. Gasparetto ${ }^{4}$
}

Paraganglioma jugulotympanic (PJT) are originated by extension of paranglioma jugulare along the course of Jacobson`s nerve and are composed by cells of the extra-adrenal neuroendocrine system ${ }^{1,2}$. Acquired cholesteatomas are non-neoplastic keratinising lesions filled by desquamation debris seen more commonly in the temporal bone that may develop from various mechanisms ${ }^{3,4}$. The association between these entities is extremely rare and to the best of our knowledge, only one previous series mentioned the development of cholesteatoma in the postoperative of paraganglioma 5 .

We report a case of cholesteatoma presenting as a late complication of PJT resection, emphasizing the importance of diffusion-weighted magnetic resonance (MR) imaging (DWI) for the diagnosis of this rare association.

\section{CASE}

A 45-year-old woman presented with a four-week history of purulent discharge with rays of blood in the left retroauricular region. Four years earlier she had a left radical mastoidectomy for total resection of a PJT, with closure by Rambo technique. Also, during the last year she had two episodes of left mastoiditis that required surgical drainage, performed out of our institution. The physical examination revealed a retroauricular fistula and peripheral facial palsy in the left side, both related to the previous procedure.

The high-resolution computed tomog-

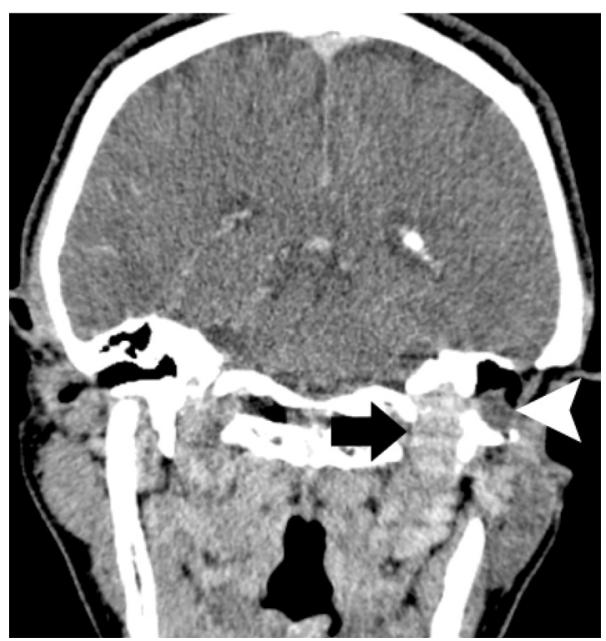

Fig 1. Contrast-enhanced coronal HRCT scan shows an intensely enhancing mass (arrow), extending to the middle ear and producing erosion of left jugular foramen. In addition, a nonenhancing soft tissue lesion was seen in the surgical cavity (arrowhead).

raphy (HRCT) scan showed a well-defined soft tissue mass with contrast-enhancement in the left jugular foramen with extension to the temporal bone and below to the skull base, producing erosion and expansion of the jugular foramen. In addition, a non-enhancing soft tissue lesion was seen in the surgical cavity, in close contact with the enhancing mass (Fig 1).

The MRI corroborated the HRCT scan findings, demonstrating a heterogeneous left jugular foramen lesion, extending to the middle ear, with low signal intensity on T1-weighted images, iso/high signal intensity on T2-weighted images, present-
Hospital Universitário Clementino Fraga Filho / UFRJ

Rua Prof. Rodolpho Paulo Rocco, 255, Departamento de Radiologia / Subsolo 21941-617 Rio de Janeiro RJ - Brasil E-mail: cgmelo09@yahoo.com.br

Received 17 February 2009 Received in final form 3 August 2009 Accepted 8 September 2009

\section{COLESTEATOMA COMO COMPLICAÇÃO TARDIA DE CIRURGIA DE PARAGANGLIOMA: O PAPEL DA RESSONÂNCIA MAGNÉTICA COM DIFUSÃO}

Departaments of Radiology and Otorhinolaryngology, Federal University of Rio de Janeiro School of Medicine, Rio de Janeiro RJ, Brazil: ${ }^{1} \mathrm{MD}$, Resident of the Department of Radiology; ${ }^{2} \mathrm{MD}$, Resident of Department of Otorhinolaringology; ${ }^{3} \mathrm{MD}$, Staff of the Department of Otorhinolaringology; ${ }^{4} \mathrm{MD}$, PhD, Associated Professor of the Department of Radiology. 


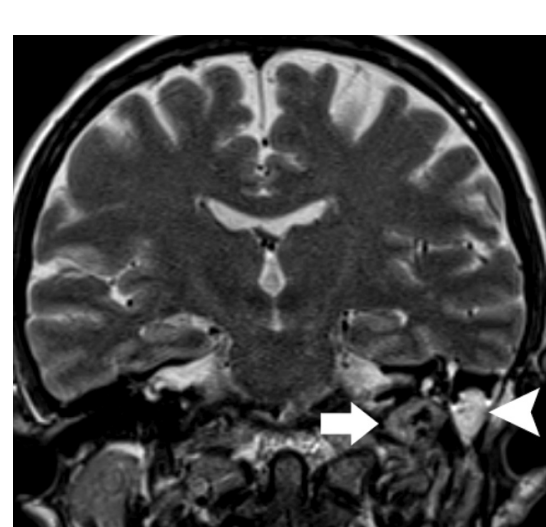

Fig 2. Coronal T2- weighted MR image demonstrates an isointensive lesion with flowvoids in the left jugular fossa and middle ear (arrow), compatible with residual PJT. Another lesion with hyperintensive signal could be seen into the surgical cavity (arrowhead). Note the close relation between them.

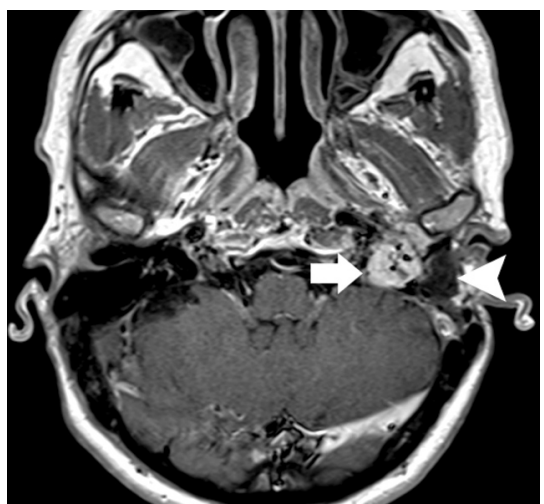

Fig 3. Axial post-gadolinium T1-weighted MR image shows the intense enhancement of the lesion at left jugular fossa/middle ear, presenting the characteristics flow voids (arrow). Notice also the non-enhancing cholesteatoma (arrowhead) in close contact with the PJT.

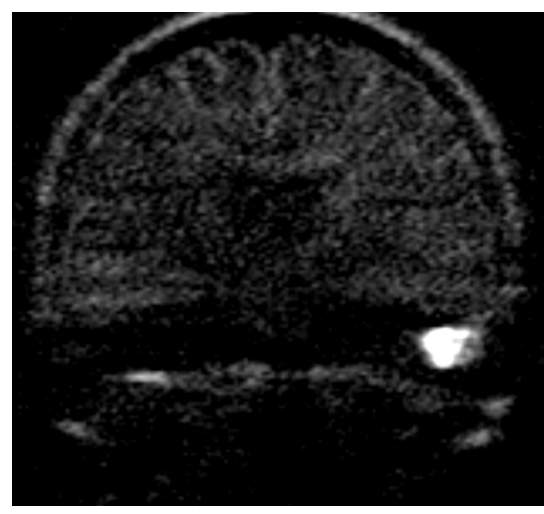

Fig 4. Coronal SS TSE DWI shows an oval hyperintensive lesion lateral to the signal void of the left middle ear, compatible with cholesteatoma. ing flow-voids within the mass and intense contrast-enhancement, measuring $1.9 \times 1.5 \mathrm{~cm}$. The non-enhancing lesion seen in HRCT scan demonstrated at MRI hypointense signal intensity on T1-weighted sequences, hyperintense signal intensity on T2-weighted images, with no enhancement after intravenous gadolinium administration, measuring $1.5 \times 1.1 \mathrm{~cm}$ (Figs 2 and 3). In the coronal single-shot turbo spin- echo (SS TSE) DWI, this lesion clearly exhibited a hyperintense signal (Fig 4). The diagnosis of residual PJT associated with cholesteatoma was suggested.

The patient underwent a revision mastoidectomy, for the resection of the cholesteatoma, and the histological study confirmed this diagnosis. Then, she was referred to the Oncology Department for treatment of the residual PJT with radiotherapy. At that time she signed informed consent allowing the publication of the case report.

\section{DISCUSSION}

Paragangliomas are extremely rare, accounting for 6\% of all neoplasm in the head and neck region. The imaging aspects allow the diagnosis with confidence. The HRCT scan is important at the diagnosis to evaluate the extension of the lesion, mainly related to the skull base. PJT appears as locally aggressive and destructive neoplastic lesion of foramen jugular and middle ear, with intense contrast enhancement. MRI provides better definition of extension and characterization of PJT and also is superior to demonstrate involvement of neural and vascular structures. At MRI, they have a low intensity signal on T1-weighted images, a high intensity signal on T2weighted images and enhance strongly after contrast. In addition, demonstrate focal intratumoral hemorrhages or slow- flow appearing as high signal intensity and flow voids produced by high-flow blood vessels, mainly in lesion bigger than $1 \mathrm{~cm}$, producing a characteristic "salt and pepper" appearance in both $\mathrm{T} 1$ and $\mathrm{T} 2$ weighted images $(1,2)$. Corroborating these findings, in our case, the MRI showed a heterogeneous mass with low signal intensity on T1-weighted images, iso/high signal intensity on T2-weighted images, presenting flow-voids and contrastenhancement.

In most cases of PJT, surgical resection of the tumor is the therapy of choice. However, even recent surgical series demonstrate a high incidence of incomplete resection, because of their highly vascular structure and their extremely critical location. Unfortunately, the spectrum of associated morbidity remains considerable, including deficits of the $\mathrm{IX}^{\text {th }}$ to XII ${ }^{\text {th }}$ cranial nerves, paralyzed vocal cords, facial nerve palsy and hearing deterioration or loss, cerebrospinal fluid leaks and so on ${ }^{5,6}$.

The development of cholesteatoma in the postoperative of paraganglioma cavity is a rare complication, and only one case was previously mentioned in the literature ${ }^{5}$. The origin of acquired cholesteatomas in a post-surgical context is uncertain, and is supported by iatrogenic, post-traumatic or implantation theories, that considers the possibility of iatrogenic implantation of epithelial cells into the middle ear, as after non-cholesteatomatous conditions or injury to the temporal bone $e^{3,4}$. We believed that the use of Rambo procedure, in witch abdominal wall fat is placed into the surgical cavity, or unsatisfactory closure of the new cavity, might incidentally have coursed with implantation of skin cells into the surgical cavity and contributed to the development of cholesteatoma.

In patients with cholesteatoma, the HRCT remains the imaging technique of choice for the diagnosis and surgical planning. They appear as a soft- tissue mass usually 
associated with erosion of bone components of the middle ear. In the MRI, they present with iso / hipointense signal intensity on T1-weighted images and moderately hyperintense signal intensity on T2-weighted images, with or without peripheral enhancement after gadolinium administration. Recently, the DWI has become the use of MRI fundamental in diagnosis of cholesteatoma and presecond look recidivate cholesteatoma ${ }^{7-10}$.

The cholesteatoma appears as a marked hyperintense lesion at DWI with no false-positives reported in the literature. The sensitivity of SS TSE DWI in detection of cholesteatoma is higher compared with other DWI sequences, such as echo-planar imaging (EPI), being able to detect lesions as small as $2 \mathrm{~mm}^{9-10}$. The exact cause of the hyperintensity signal in cholesteatomas is not completely understood, but most of the studies attributed this abnormality to $\mathrm{T} 2$ shine-through effect ${ }^{7-10}$. In our case, the SS TSE DWI was essential for the diagnosis of cholesteatoma in a surgical cavity of a previous resected PJT.

In summary the development of cholesteatoma in the surgical cavity of a post-operative paraganglioma is extremely rare. In the present report, the use of SS TSE DWI was essential for the diagnosis of this unusual complication of PJT surgery, explaining the reason of the pa- tient symptoms and helping the planning of the best therapeutic option.

\section{REFERENCES}

1. Rao AB, Koeller KK, Adair CF. Paragangliomas of head and neck: radiologicpathologic correlation. Radiographics 1999;19:1605-1632.

2. Mattos LP, Ramina R, Borges W, et al. Intradural jugular foramen tumors. Arq Neuropsiquiatr 2004,62:997-1003.

3. Persaud R, Hajioff D, Trinidade A, et al. Evidence-based review of aetiopathogenic theories of congenital and acquired cholestetoma. J Laryngol Otol 2007;121:1013-1019.

4. Youngs R. Epithelial migration in open mastoidectomy cavities. J Laryngol Otol 1995;109:286-290.

5. Forest JA, Jackson CG, McGrew BM. Long-term control of surgically treated glomus tympanicum tumors. Otol Neurol 2001; 22:232-236.

6. Gerosa M,Visca A, Rizzo P, Foroni R, Nicolato A, Bricolo A. Glomus jugulare tumors: the option of gamma knife radiosurgery. Neurosurgery 2006;59:561-569.

7. Vercruysse JP, De Foer B, Pouillon M, Somers T, Casselman JW, Offeciers E. The value of diffusion-weighted MR imaging in the diagnosis of primary acquired and residual cholesteatoma: a surgical verified study of 100 patients. Eur Radiol 2006;16:1461-1467.

8. Aikele P, Kittner T, Offergeld C, Kaftan H, Huttenbrink KB, Laniado M. Diffusionweight MR imaging of cholesteatoma in pediatric and adult patients who have undergone middle ear surgery. Am J Roentgenol 2003;181:261-265.

9. De Foer B, Vercruysse JP, Bernaerts A, et al. The value of single-shot turbo spin-echo diffusion-weight MR imaging in the detection of middle ear cholesteatoma. Neuroradiology 2007;49:841-848.

10. Vercruysse JP, Pilet B, Michiels J, et al. Single-shot, turbo spin-echo, diffusion-weighted imaging versus spin-echo-planar, diffusion-weighted imaging in the detection of acquired middle ear cholesteatoma. Am J Neuroradiol 2006;27:1480-1482. 